\title{
The EGFR pathway is involved in the regulation of PD-L1 expression via the IL-6/JAK/STAT3 signaling pathway in EGFR-mutated non-small cell lung cancer
}

\author{
NAN ZHANG ${ }^{1 *}$, YUANYUAN ZENG $^{1,2^{*}}$, WENWEN DU ${ }^{1 *}$, \\ JIANJIE ZHU ${ }^{1,2}$, DAN SHEN ${ }^{1}$, ZEYI LIU ${ }^{1,2}$ and JIAN-AN HUANG ${ }^{1,2}$ \\ ${ }^{1}$ Department of Respiratory Medicine, The First Affiliated Hospital of Soochow University, Suzhou, Jiangsu 215006; \\ ${ }^{2}$ Institute of Respiratory Diseases, Soochow University, Suzhou, Jiangsu 215006, P.R. China
}

Received May 13, 2016; Accepted June 15, 2016

DOI: $10.3892 /$ ijo.2016.3632

\begin{abstract}
Negative regulation of the signal mediated by the programmed cell death protein 1 (PD-1)/programmed deathligand 1 (PD-L1) pathway can effectively inhibit the function of $\mathrm{T}$ and $\mathrm{B}$ cells, which play a key role in the regulation of immune response. Recently, emerging evidence has suggested that the expression of PD-L1 is related to the mutation status of the epidermal growth factor receptor (EGFR). Moreover, the activation of the EGFR signaling pathway can induce expression of PD-L1. In the present study, we demonstrated that activated EGFR can upregulate the expression of PD-L1 through the interleukin 6/Janus kinase/signal transducer and activator of transcription 3 (IL-6/JAK/STAT3) signaling pathway in non-small cell lung cancer (NSCLC) cells. Cells treated with epidermal growth factor receptor tyrosine kinase inhibitors (EGFR-TKIs) can downregulate the activation of the IL-6/JAK/STAT3 pathway, which subsequently reduces the expression of PD-L1. Furthermore, silencing of PD-L1 expression in NSCLC cells correlated with inhibition of cell proliferation and enhanced tumor cell apoptosis. In summary, our research indicates that EGFR is involved in the regulation of PD-L1 expression and cell proliferation via the IL-6/JAK/STAT3 signaling pathway in NSCLC. The present study suggests the potential of combined targeted therapy with immunotherapy in the treatment of NSCLC.
\end{abstract}

\section{Introduction}

Lung cancer is one of the most common types of cancer and is the leading cause of cancer death worldwide (1). Approximately

Correspondence to: Professor Jian-An Huang, Department of Respiratory Medicine, the First Affiliated Hospital of Soochow University, Suzhou, Jiangsu 215006, P.R. China

E-mail: huang_jian_an@163.com

*Contributed equally

Key words: epidermal growth factor receptor, non-small cell lung cancer, programmed death-ligand 1, IL-6, JAK, STAT3
80-85\% of lung cancer is non-small cell lung cancer (NSCLC) and disease is detected in advanced stages in more than $65 \%$ of NSCLC patients $(2,3)$. However, owing to the development of platinum-based chemotherapy and operation methods, the outcome of NSCLC has significantly improved. Nevertheless, NSCLC is still an increasing threat to human health.

Recent studies in human genetics have led to a new strategy of cancer treatment: personalized targeted therapy based on the status of oncogenic drivers mutated in tumor cells $(4,5)$. To date, small molecular drugs targeting the epidermal growth factor receptor (EGFR) and anaplastic lymphoma kinase (ALK) have been widely used in NCSLC patients who have EGFR or ALK mutations, respectively. Among all of these treatments, the epidermal growth factor receptor tyrosine kinase inhibitor (EGFR-TKI) stands out as one of the most effective medicines for patients with NSCLC, as it significantly prolongs overall survival (OS) and progression-free survival (PFS) (5-7).

Recent studies have suggested that activation of EGFR is positively associated with the activation of PD-L1 in NSCLC cells. EGFR mutations are directly related to the upregulation of PD-L1 expression (8-11). Programmed cell death protein 1 (PD-1) and its ligand the PD-L1, play a major role in suppressing the immune system during cancer development and can therefore be used as immunotherapy target. Some in vivo studies have shown that blocking the PD-1/PD-L1 pathway can restore the antitumor activity of $\mathrm{T}$ cells $(12,13)$. Furthermore, in clinical trials, PD-1/PD-L1 checkpoint inhibitors showed tolerable efficacy in patients with advanced or metastatic NSCLC (14). All of these results indicate that highly activated EGFR is associated with overactivation of the PD-1/ PD-L1 pathway, which leads to an increasing risk of tumor immune escape in NSCLC. However, the potential mechanism behind this event remains unclear.

One recent study indicated that interleukin 6 (IL-6)mediated PD-L1 expression in tolerogenic antigen-presenting cells (APCs) was controlled by signal transducer and activator of transcription 3 (STAT3). More specifically, activated STAT3 was shown to bind to the PD-L1 promoter and, subsequently, promote transcription of PD-L1 (15). To the best of our knowledge, the IL-6/Janus kinase (JAK)/STAT3 is a canonical signaling pathway which plays a critical role 
in the initiation, development and formation of various cancers (16). One previous study suggested that the EGFR signaling pathway is involved in the activation of the IL-6/ JAK/STAT3 pathway (17). Taken together, it is tempting to speculate that EGFR can regulate the expression of PD-L1 via the IL-6/JAK/STAT3 pathway.

In the present study, we investigated the relationship between the EFGR signaling pathway and PD-L1 in NSCLC cells and the involvement of IL-6/JAK/STAT3 pathway in this relationship. Furthermore, we also evaluated the role of PD-L1 in survival of NSCLC cells.

\section{Materials and methods}

Cell culture, treatment and transfection. Human EGFR wild-type NSCLC cell lines (i.e. H1299 and SPC-A1) and EGFR-mutated NSCLC cell lines (i.e. H1975 and HCC827) were obtained from the Shanghai Institutes of Biological Sciences Cell Bank and were maintained in RPMI-1640 medium (Gibco) supplemented with $10 \%$ fetal bovine serum (FBS; Gibco) and 1\% penicillin/streptomycin (Gibco). PC-9 cells were obtained from Professor Cai-cun Zhou as a gift and were maintained in Dulbecco's modified Eagle's medium (DMEM; Gibco) supplemented with 10\% FBS (Gibco) and $1 \%$ penicillin/streptomycin (Gibco). All cells were cultured in humidified incubators at $37^{\circ} \mathrm{C}$ with $5 \% \mathrm{CO}_{2}$.

For gefitinib treatment, cells were seeded into 96-well plates at a density of $3 \times 10^{3}$ cells/well, grown overnight and then induced with varying drug concentrations for $48 \mathrm{~h}$ : $0,0.005$, $0.01,0.1,1,5,10,20$ and $40 \mu \mathrm{M}$ gefitinib (AstraZeneca). For AG490 treatment, cells were seeded into 6 -well plates $\left(3 \times 10^{5}\right.$ cells/well), grown overnight and then serum-starved for $24 \mathrm{~h}$. Subsequently, serum-starved cells were treated with AG490 (100 $\mu \mathrm{M}$; Sigma-Aldrich) for $4 \mathrm{~h}$.

For small interfering RNA (siRNA) transfection, predesigned siRNA targeting PD-L1 and STAT3 were purchased from Shanghai GenePharama Co., Ltd., Shanghai, China. Transfections were performed using the siRNA transfection kit (Shanghai GenePharama) according to the manufacturer's protocol.

EGFR mutation analysis. Genomic DNA samples were extracted by the Genomic DNA kit (Tiangen Biotech Co., Ltd., Beijing, China) according to the manufacturer's instructions and sent to our collaborator in the Central Laboratory of the First Affiliated Hospital of Soochow University for EGFR mutation analysis (exons 18-21) by amplification-refractory mutation system (ARMS) PCR and direct sequencing.

Enzyme-linked immunosorbent assay (ELISA). Cell-free supernatant was harvested after treatment at different timepoints, i.e. $0,2,4,8,12,24$ and 48 h. IL-6 ELISA analyses were conducted by using a commercially available ELISA kits (Multi Sciences) according to the protocols suggested by the manufacturer. Standard dilutions were prepared as suggested. The OD450 was read and the concentration of IL-6 was calculated according to the standard curve.

Western blot analysis. Approximately $10 \times 10^{6}$ cells were harvested and lysed in 1X RIPA buffer with proteinase and phosphatase inhibitors. Cell lysates were cleared by centrifugation at $4^{\circ} \mathrm{C}$ for $20 \mathrm{~min}$ at $12000 \mathrm{x} \mathrm{g}$. Protein was separated by sodium dodecyl sulfate-polyacrylamide gel electrophoresis (SDS-PAGE) and transferred onto a polyvinylidene fluoride (PVDF) membrane. After blocking in 5\% non-fat milk/T-BST buffer for $1 \mathrm{~h}$, membranes were incubated in primary antibodies overnight at $4^{\circ} \mathrm{C}$. The following primary antibodies were used in the dilution as suggested by the supplier: PD-L1 (1:1,000; Cell Signal Technology), p-EGFR (1:1,000; Cell Signal Technology), p-STAT3 (1:1,000; Cell Signal Technology), STAT3 (1:1,000; Cell Signal Technology), GAPDH (1:4,000; Cell Signal Technology). After primary antibody incubation, blots were incubated in HRP-conjugated secondary antibodies for 2-3 $\mathrm{h}$ at room temperature. Immunoreactive proteins were detected using the SuperSignal West Pico Chemiluminescent substrate (Thermo Fisher Scientific).

$R N A$ extraction and real-time polymerase chain reaction $(R T-P C R)$. Total RNA was extracted from cells using the TRizol method (Takara Bio). Synthesis of cDNA with reverse transcriptase was performed with the M-MLV First Strand kit (Invitrogen). cDNA aliquots were subjected to RT-PCR reactions using the SYBR Premix Ex Tag II (Takara) on the ABI PRISM 7500 Sequence Detection system (Applied Biosystems). Primers used for RT-PCR were as follows: 5'-GGTGCCGA CTACAAGCGAAT-3' (forward) and 5'-GGTGACTGGATC CACAACCAA-3' (reverse) for PD-L1; and 5'-TGCACCACC AACTGCTTAGC-3' (forward) and 5'-GGCATGGACTGTG GTCATGAG-3' (reverse) for GAPDH.

Cell proliferation assay. Cells were seeded in 96-well plates at a density of $3 \times 10^{3}$ cells/well. After 24,48 and $72 \mathrm{~h}$, every well was treated with $10 \mu \mathrm{l}$ of cell Counting kit-8 solution (Beyotime Institute of Biotechnology) and the OD450 was measured by a microplate reader after $4 \mathrm{~h}$ (Thermo Fisher Scientific).

Colony formation assay. Cells were seeded ( $3 \times 10^{3}$ cells/well) in a 6-cm culture dish. After a week, cells were washed twice with PBS, fixed with methanol for 30 min and stained with crystal violet overnight. Images from every plate were taken and analyzed by Image $J$ software.

Cell apoptosis analyses. Cell apoptosis was detected using an Annexin V-fluorescein isothiocyanate (FITC)/propidium iodide (PI) apoptosis detection kit (Beyotime Institute of Biotechnology) according to the manufacturer's instructions. Briefly, cells were washed twice with cold PBS and then $5 \times 10^{4}$ cells were resuspended in $1 \mathrm{ml}$ binding buffer containing Annexin V/FITC and PI. After incubation at room temperature for $20 \mathrm{~min}$ in the dark, staining results were detected by the fluorescence-activated cell sorting FACSCaliber system (Beckman Coulter) within 30 min.

Statistical analysis. All numerical data were presented as the mean \pm standard error of the mean (SEM). Statistical analysis was performed by a two-tailed Student's t-test and the difference was considered significant at P-value of $<0.05$. The statistical analyses were carried out with SPSS 17.0 software. 
$\mathbf{A}$
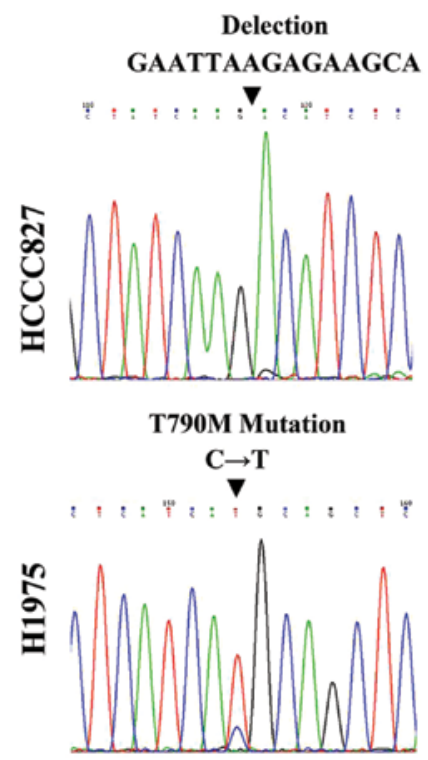

B

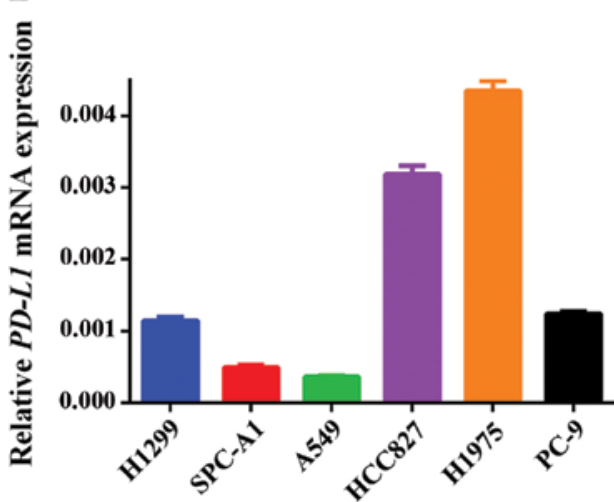

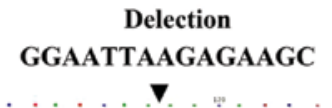

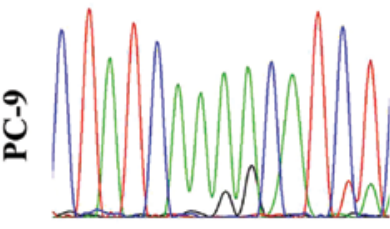

L858R Mutation
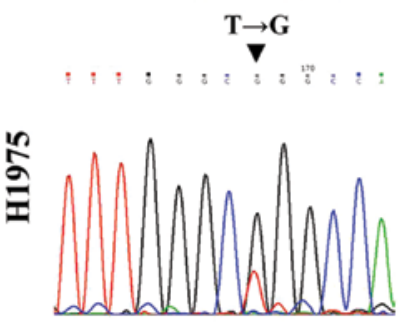

Figure 1. The expression of PD-L1 is closely related with EGFR mutations. (A) By sequencing, the mutation points of the EGFR-mutant cell lines (HCC827, PC-9 and H1975) were confirmed. (B) The mRNA and protein expression level of PD-L1 were detected by RT-PCR and western blot analysis, respectively in H1299, SPC-A1, A549, HCC827, H1975 and PC-9 cells. GAPDH was used as a loading control in western blot analysis.

\section{Results}

The expression of PD-L1 is closely related with EGFR mutations. The variation in EGFR exon 18-21 was evaluated in three EGFR-mutant cell lines, i.e. HCC827, PC-9 and H1975. An activating deletion in exon 19 was found in the HCC827 and PC-9 cell lines and a leucine-to-arginine substitution at codon 858 (p.L858R) together with a threonine-to-methionine substitution at codon 790 (p.T790M) were detected in the H1975 cell line (Fig. 1A).

The transcription and translation level of PD-L1 was measured by RT-PCR and western blot analysis in three EGFR-mutant cell lines as mentioned above and three EGFR wild-type cell lines, i.e. H1299, SPC-A1 and A549. As shown in Fig. 1B, PD-L1 expression was much higher in the three EGFR-mutant cell lines in comparison to the EGFR wild-type cell lines, with the exception of the H1299 cell line, which had comparable PD-L1 expression levels as seen in the PC-9 cells (Fig. 1B). The potential reason for this could be due to the deletion of TP53 in these cells, which has already be shown to be closely related with PD-L1 expression (18).

In summary, we were able to associate the expression of PD-L1 with EGFR mutations in NSCLC cells. EGFR- activating mutations can upregulate the transcription and translation of PD-L1.

EGFR signaling pathway is involved in the regulation of $P D-L 1$ expression in EGFR-mutant NSCLC cells. To investigate the relationship between EGFR activation and PD-L1 expression in EGFR-mutant NSCLC cells, we induced HCC827 and PC-9 cells with the recombined human epidermal growth factor (EGF) in various concentrations $(0,25,50$ and $75 \mathrm{ng} / \mathrm{ml})$ for $48 \mathrm{~h}$ in order to activate the EGFR pathway. The transcriptional change of PD-L1 was measured by RT-PCR, which showed a positive association between EGF concentration and PD-L1 mRNA levels (Fig. 2A). The PD-L1 protein expression change was compared between untreated and EGF-treated $(50 \mathrm{ng} / \mathrm{ml})$ cells (HCC827 and PC-9) by western blot analysis, which indicated a significant increase after treatment (Fig. 2B). All of these results suggested that EGFR activation led to PD-L1 upregulation.

For more clarification, we treated HCC827 and PC-9 cells with gefitinib (an EGFR-TKI) in different concentrations according to the half maximal inhibitory concentration $\left(\mathrm{IC}_{50}\right)$ which we obtained from our preliminary experiments (data not shown) so that we could inhibit the activation of the EGFR 

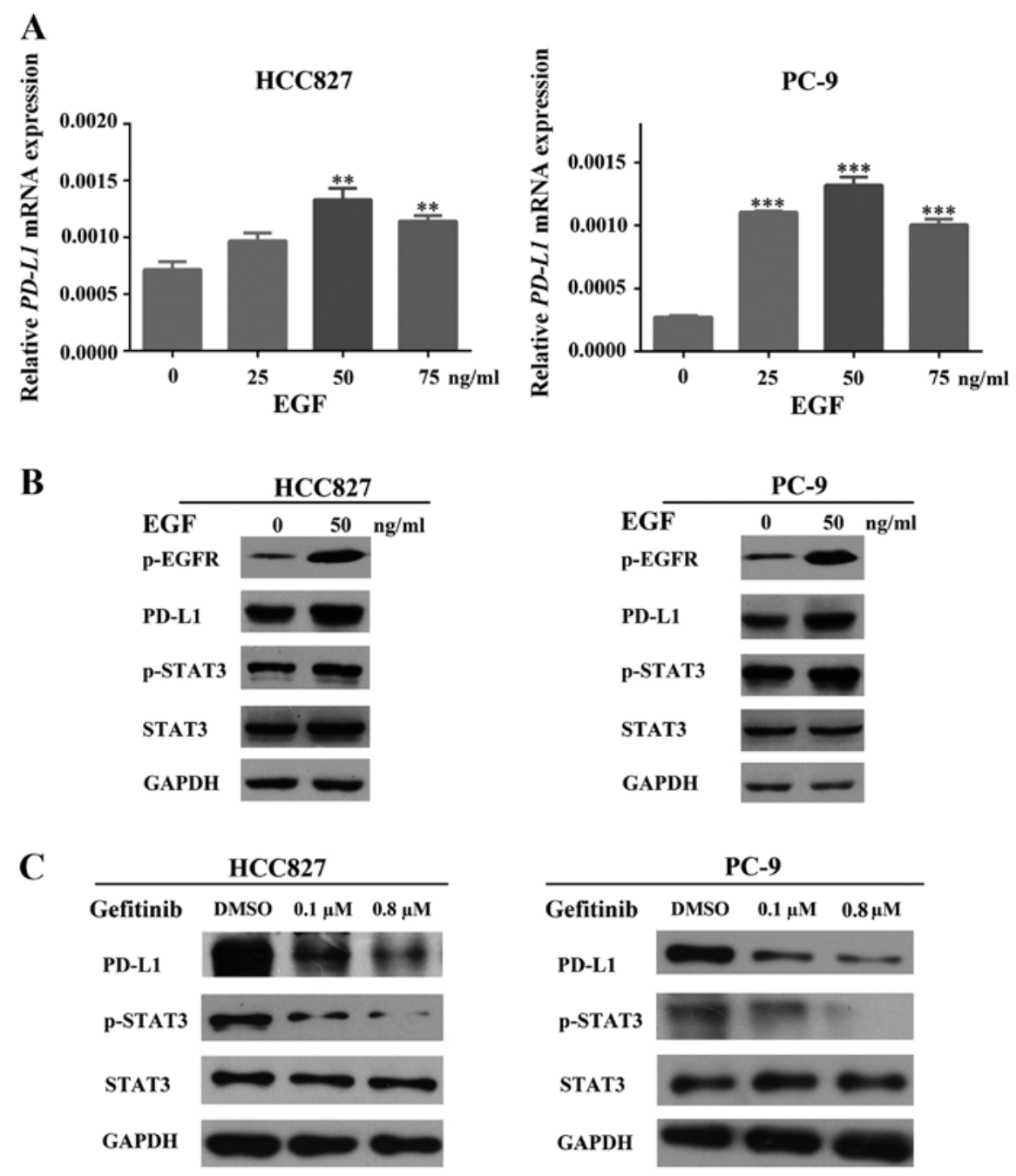

Figure 2. EGFR signaling pathway is involved in the regulation of PD-L1 expression in EGFR mutant NSCLC cells. (A) HCC827 and PC-9 cells were treated with different dose $(0,25,50$ and $75 \mathrm{ng} / \mathrm{ml})$ of EGF for $48 \mathrm{~h}$. The expression of PD-L1 at mRNA level was detected by RT-PCR. (B) HCC 827 and PC-9 cells were stimulated with $(50 \mathrm{ng} / \mathrm{ml})$ or without EGF for $48 \mathrm{~h}$, the expression of p-EGFR, PD-L1, p-STAT3 and STAT3 were detected by the western blot analysis. GAPDH was used as a loading control. (C) HCC 827 and PC-9 cells were stimulated with 0.1 and $0.8 \mu \mathrm{M}$ or without gefitinib for $48 \mathrm{~h}$, the expression of PD-L1, p-STAT3 and STAT3 were detected by the western blot analysis. GAPDH was used as a loading control. $\left({ }^{* *} \mathrm{P}<0.01,{ }^{* * *} \mathrm{P}<0.001\right)$.

pathway. The expression of PD-L1 was measured by western blot analysis, which suggested a significant dose-dependent decrease of PD-L1 presenting after gefitinib treatment (Fig. 2C).

As a result, activity of EGFR is positively related with the expression of PD-L1 in EGFR-mutant NSCLC.

IL-6/JAK/STAT3 signaling pathway is involved in regulation of EGFR-mediated PD-L1 expression in EGFR-mutant NSCLC cells. As described previously, IL-6/JAK/STAT3 is considered to be a potential pathway involved in the regulation of EGFR-mediated PD-L1 expression. To confirm this, we investigated the activation of STAT3 in samples from previous EGFR activation and inhibition experiments. As shown in Fig. 2B and C, HCC827 and PC-9 cells induced with EGF were able to increase the phosphorylation and activation of EGFR and STAT3. However, inhibiting the activation of EGFR by gefitinib was able to dephosphorylate and deactivate STAT3. Moreover, we also checked the IL- 6 levels in cell-free supernatants from gefitinib-treated $(0.8 \mu \mathrm{M}) \mathrm{HCC} 827$ and PC-9 cells via ELISA at different time-points $(0,2,4,8,12,24$ and
$48 \mathrm{~h})$. In both untreated and gefitinib treated cells, the concentration of IL-6 increased with time. However, this increase was significantly slower in gefitinib-treated cells $(\mathrm{P}<0.01$; Fig. $3 \mathrm{~A})$.

To further confirm these results, we silenced the endogenous expression of STAT3 and assessed the expression level of PD-L1 in HCC827 and PC-9 cells. As shown in Fig. 3C, depletion of STAT3 expression induced a significant loss of PD-L1 at both protein and mRNA levels $(\mathrm{P}<0.01)$.

All of this evidence indicated that EGFR activation can induce the expression and secretion of IL-6 from NSCLC cells, which conversely increases the activation of STAT3. Activated STAT3 eventually leads to the increasing expression of PD-L1.

To investigate the involvement of JAK, we treated HCC 827 and PC-9 cells with AG $490(100 \mu \mathrm{M})$, which is a specific and potent JAK-2 protein tyrosine kinase inhibitor. After $4 \mathrm{~h}$ of treatment, the amount of PD-L1 protein and mRNA was measured and results indicated a significant reduction in both levels $(\mathrm{P}<0.001$; Fig. 3B).

The above-mentioned results suggest that EGFR can mediate PD-L1 expression in EGFR-mutant NSCLC cells by upregulating the expression of IL-6 which, subsequently, 


\section{$\mathbf{A}$}

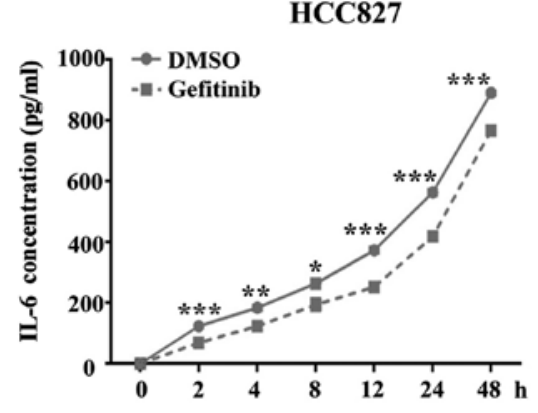

PC-9

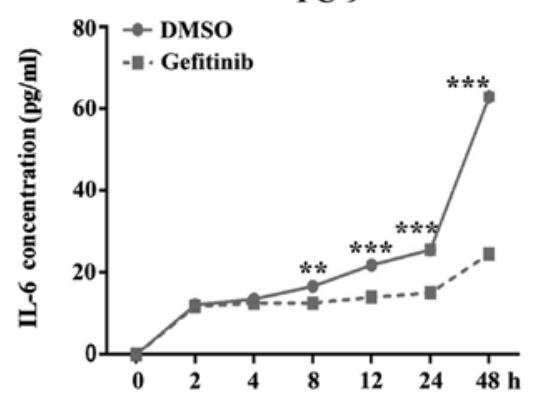

B

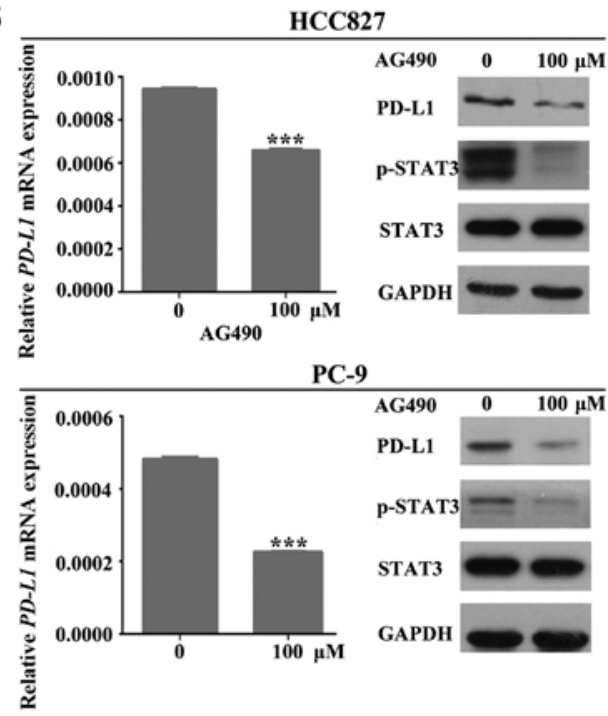

C
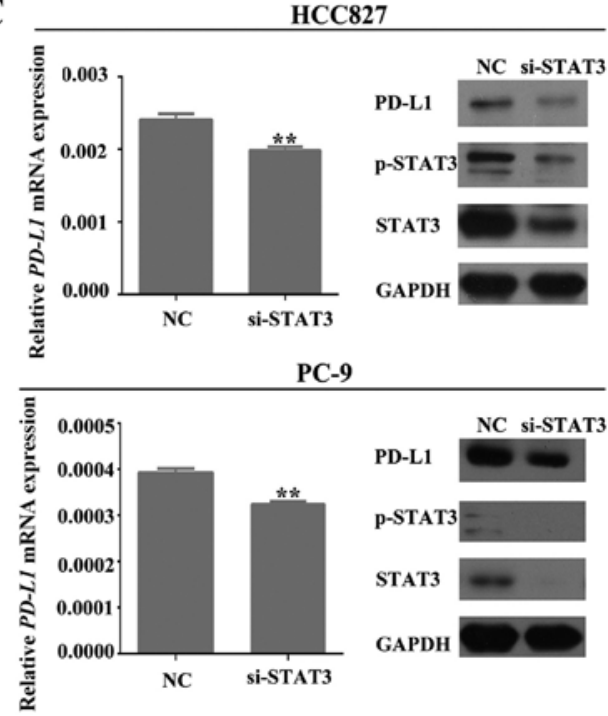

Figure 3. IL-6/JAK/STAT3 signaling pathway is involved the regulation of EGFR mediated PD-L1 expression in EGFR mutant NSCLC cells. (A) HCC827 and PC-9 cells were stimulated with gefitinib $(0.8 \mu \mathrm{M})$ or without gefitinib for $48 \mathrm{~h}$. The cell-free supernatants were harvested at different time-points. The concentration of IL- 6 was determined by ELISA. (B) HCC 827 and PC-9 cells were stimulated with (100 $\mu$ M) or without AG490 for 48 h. The expression of PD-L1, STAT3 and p-STAT3 were detected by western blot analysis. GAPDH was used as a loading control. (C) The endogenous expression of STAT3 was silenced by siRNA in HCC827 and PC-9 cells. Expression of PD-L1, p-STAT3 and STAT3 were detected by western blot analysis. GAPDH was used as a loading control. $\left({ }^{*} \mathrm{P}<0.05 ;{ }^{* *} \mathrm{P}<0.01 ;{ }^{* * *} \mathrm{P}<0.001\right)$.

activates the IL-6/JAK/STAT3 signaling pathway. Activated STAT3 can eventually increase the expression of PD-L1 in EGFR-mutant NSCLC cells.

PD-L1 plays a role in cell survival in EGFR-mutant NSCLC cells. To predict the potential role of PD-L1 in cell survival, we silenced PD-L1 expression in HCC827 and PC-9 cells and then investigated the changes in cell proliferation and apoptosis. The efficacy of silencing was confirmed by western blot analysis and RT-PCR (Fig. 4A). Inhibition of PD-L1 accumulation in HCC827 and PC-9 cells led to a remarkable decrease in cell proliferation $(\mathrm{P}<0.01$; Fig. 4B). Moreover, downregulation of PD-L1 expression enhanced apoptosis of HCC827 and PC-9 cells $(\mathrm{P}<0.01$; Fig. 5). As shown in Fig. 5, silencing of PD-L1 can induce cells into early apoptosis, which suggests its potential role in the prevention of apoptosis in these cells.
In summary, these data demonstrate that PD-L1 can affect tumor cell survival by promoting cell proliferation and preventing cell apoptosis, which can be reversed by downregulating the expression of PD-L1. Taking all previous results together indicates that EGFR activation can induce the expression of PD-L1 through the IL-6/JAK/STAT3 signaling pathway, which, in the end, promotes the survival of cancer cells by inhibiting apoptosis and promoting proliferation. NSCLC cells treated with EGFR-TKIs can reverse all of these effects which in turn lead to the death of tumor cells.

\section{Discussion}

As widely known, EFGR, which is a key molecule in tumor cell proliferation, invasion and metastasis regulation (19), is frequently mutated in NSCLC. EGFR-TKIs, such as gefitinib 

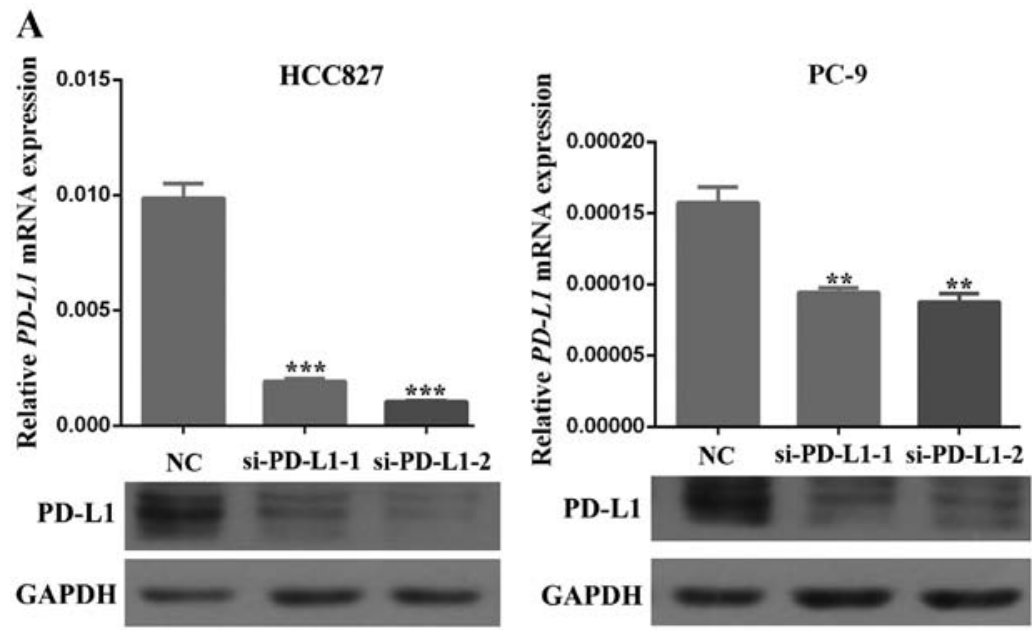

B

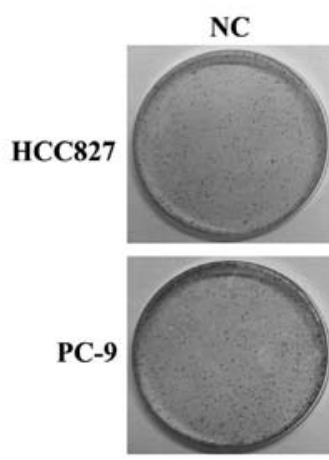

si-PD-L1-1

si-PD-L1-2
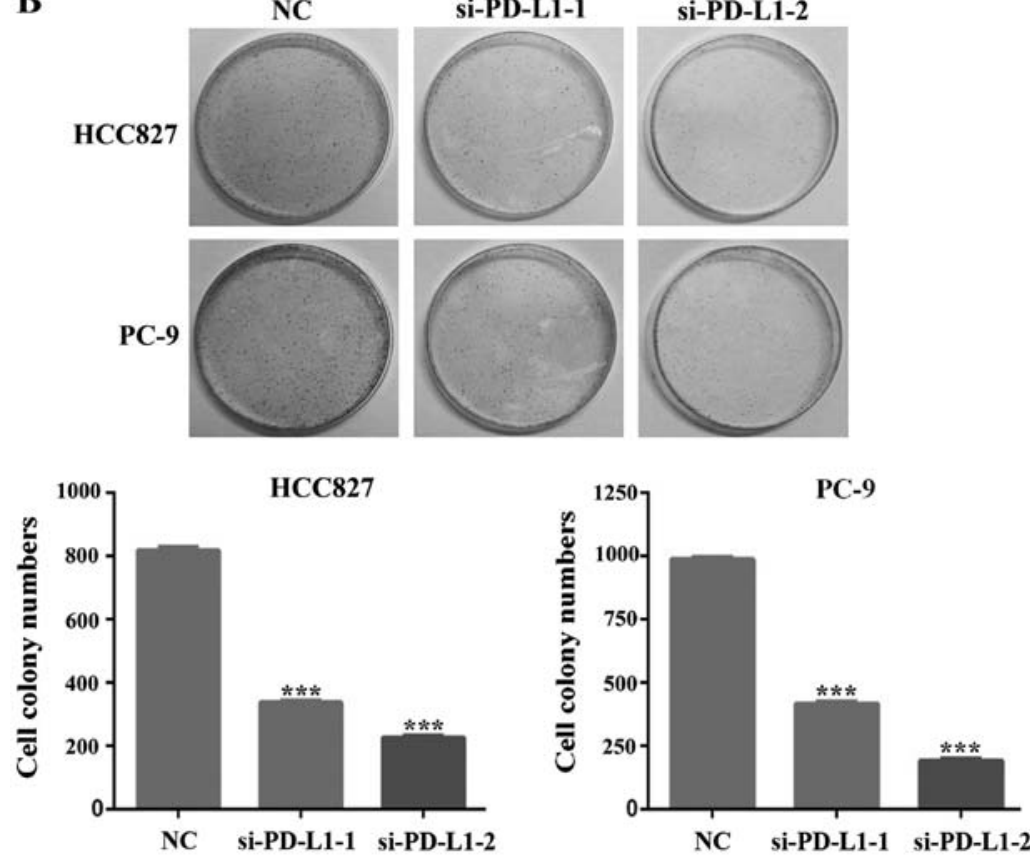

Figure 4. PD-L1 plays a role in promoting proliferation of EGFR mutant NSCLC cells. (A) The endogenous expression of PD-L1 was silenced by siRNA in HCC827 and PC-9 cells. The expression of PD-L1 was detected by western blot analysis. GAPDH was used as a loading control. The transcription of PD-L1 mRNA was detected by RT-PCR. (B) The endogenous expression of PD-L1 was silenced by siRNA in HCC827 and PC-9 cells. Cells were seeded (3x10 cells/well) in a 6 -cm culture dish. After a week, the amount of clones was analyzed by ImageJ software. $\left.{ }^{* * *} \mathrm{P}<0.01 ;{ }^{* * *} \mathrm{P}<0.001\right)$.

and erlotinib, have been approved as a first-line therapy for EGFR-mutant advanced NSCLC patients (20). Recently, increasing attention has been given to the association between EGFR-targeted therapy and immunotherapy. One focus in particular is the relationship between EGFR-TKIs and tumor cell immune evasion.

PD-L1 is a co-inhibitory molecule generally expressed in APCs (e.g. macrophages, DCs), activated T cells, B cells and tumor cells $(21,22)$. PD-L1-activated PD-1 functions as an inhibitor for the proliferation, survival and effects of $\mathrm{CD}^{+}$ cytotoxic T lymphocytes (CTLs), which play a key role in tumor immune evasion (23).

In the present study, we demonstrated that EGFR mutations are associated with increased PD-L1 expression in NSCLC cell lines. One exception was also detected: H1299, an EGFR wild-type cell line, retained relatively high expres- sion levels of PD-L1. One potential reason for this could be the deletion of the TP53 gene in these cells. It has been recently suggested that the expression of PD-L1 is regulated by p53 via miR-34, and that loss of p53 is associated with higher PD-L1 expression (18). We also found out that EGFR is involved in the regulation of PD-L1 expression via the IL-6/JAK/STAT3 signaling pathway in EGFR-mutant NSCLC. Cells induced with EGF, which activates the EGFR pathway, can upregulate the expression of PD-L1. Moreover, inhibition of EGFR by EGFR-TKIs reduced the secretion of IL-6 from cancer cells and, subsequently, decreased the activation of JAK/STAT3, which eventually inhibited the expression of PD-L1 in these cells. Some investigators also found that STAT3 promotes the expression of PD-L1 by binding to the PD-L1 promoter (15). Recently, research pertaining to $\mathrm{PD}-\mathrm{L} 1$ discovered various signaling molecules 

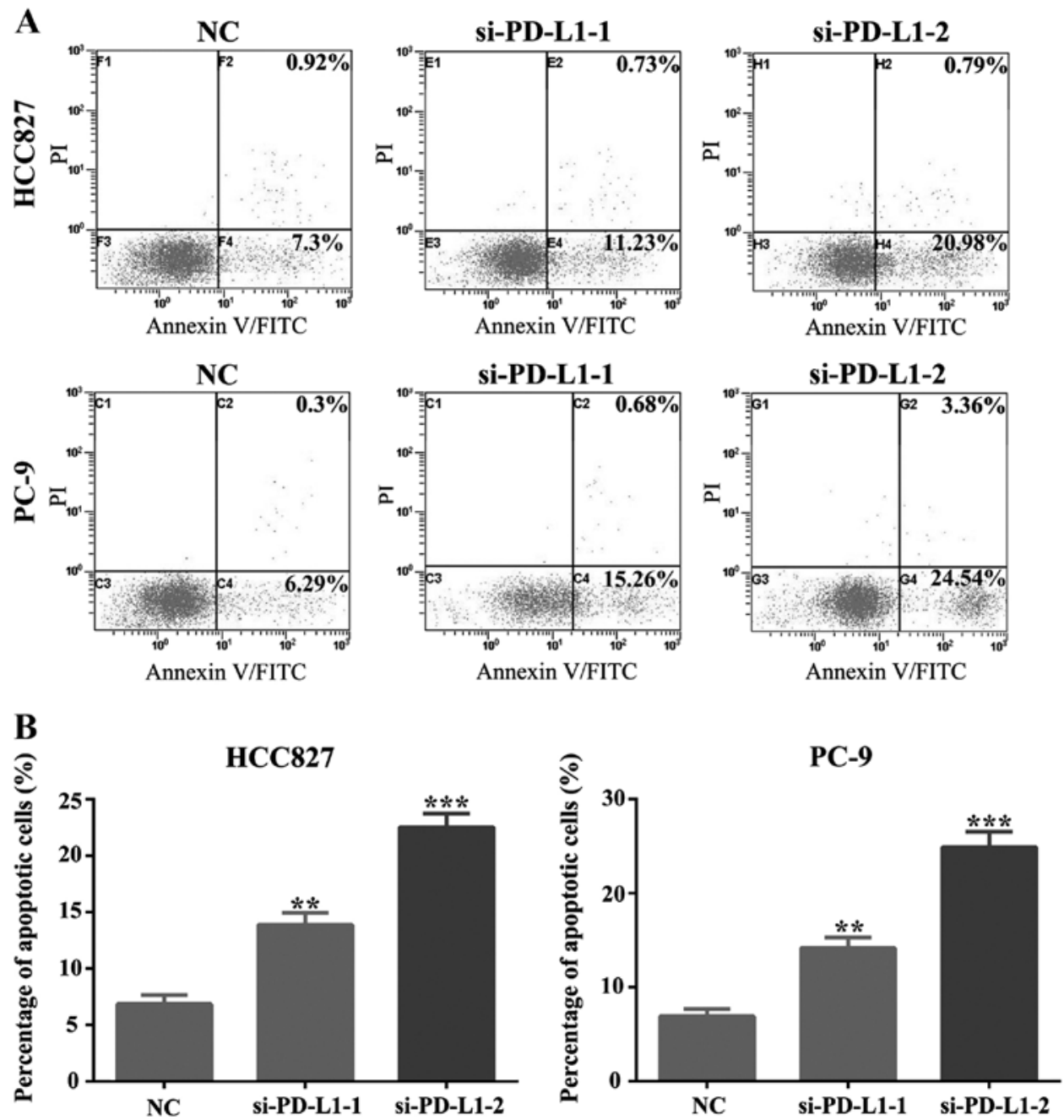

Figure 5. PD-L1 plays a role in inhibiting apoptosis in the cells. (A) The endogenous expression of PD-L1 was silenced by siRNA in HCC827 and PC-9 cells. Cells were dyed with Annexin V/PI and the apoptotic rates were measured by flow cytometric analysis. (B) The amount of apoptotic cells after silencing of PD-L1 in HCC827 and PC-9 cells. $\left({ }^{* *} \mathrm{P}<0.01 ;{ }^{* * *} \mathrm{P}<0.001\right)$.

which are potentially involved in its expression (including IFN- $\gamma$, NF- $\mathrm{BB}, \mathrm{MAPK}, \mathrm{PI} 3 \mathrm{~K} / \mathrm{AKT}$, mTOR and MEK/ERK/ STAT1) in tumor cells (24-31). NF- $\mathrm{KB}$ and MEK/ERK/STAT1 may also be important for NSCLC, as they have both been found to be involved in the regulation of EGFR-mediated PD-L1 expression. The cross-talk among all of these pathways mentioned above is an interesting area which requires further research. In the end, we were also able to show that silencing of PD-L1 can reduce proliferation and increase apoptosis in NSCLC cells. Some recent clinical studies have suggested that overexpression of PD-L1 is correlated with poor prognosis and shortened overall survival (OS) (10,3234). Together with our data, down-regulated PD-L1 might be a favorable biomarker for NSCLC.

EGFR-TKIs have already been widely applied in the clinic and are used as a first-line treatment for EGFR-mutant NSCLC patients. However, the rapid acquired resistance is still a serious limitation for targeted therapy. Based on our research, immunotherapy based on targeting PD-1/PD-L1 may bring on new approaches which are more efficient for the treatment of EGFR-mutant patients who have developed resistance to EGFR-TKIs. Our data suggest that both mutation and activation of EGFR can increase the expression of PD-L1. This means that EGFR-mutant patients who are resistant to EGFR-TKIs might also have high levels of PD-L1 present. Furthermore, in one particular phase III clinical trial, the antiPD-1 antibody (Nivolumab) was shown to increase the OS and PFS of patients with previously treated advanced or metastatic NSCLC (14). As a result, we assume that PD-1/PD-L1 targeted immunotherapy is a potent solution for EGFR-TKI resistance. Recently, the potential of combining EGFR-TKIs with immunotherapy to improve the outcome of NSCLC patients was reported (35). Targeted therapy usually has rapid and impressive response rates but modest progression-free survival, while immunotherapy can achieve durable tumor control, but is associated with lower response rates (36). Based on the present study, we demonstrate that combined EGFR-TKI and PD-1/ PD-L1-based immunotherapy may not be a good strategy, as NSCLC cells treated with EGFR-TKI can inhibit the expression of PD-L1, which in turn can significantly reduce the efficiency of the PD-1/PD-L1 inhibitor. In this case, additional immune system check points should be considered, such as CTLA4. Further research and additional clinical trials are still needed. 
In summary, the present study demonstrated that PD-L1 is overexpressed in EGFR-mutant NSCLC cells. EGFR activation can induce the expression of PD-L1 via the IL-6/JAK/STAT3 signaling pathway in EGFR-mutant NSCLC cells, which can be inhibited by EGFR-TKIs. In addition, downregulation of PD-L1 was associated with inhibited proliferation and enhanced apoptosis of EGFRmutant NSCLC cells. This study provides us with both a new perspective regarding the antitumor mechanism of EGFR-TKIs and novel thinking about the combination of EGFR-TKIs with immunotherapy.

\section{Acknowledgements}

We are grateful to Professor Caicun Zhou (Shanghai Pulmonary Hospital) for providing the PC-9 cell lines. The present study was supported by grants from the National Natural Science Foundation of China (31270940 to J.-A.H.), Clinical Medical Center of Suzhou (Szzx201502) and Clinical Key Speciality Project of China.

\section{References}

1. Siegel RL, Miller KD and Jemal A: Cancer statistics, 2015. CA Cancer J Clin 65: 5-29, 2015.

2. Reck M,Heigener DF, Mok T, Soria JC and Rabe KF: Management of non-small-cell lung cancer: Recent developments. Lancet 382: 709-719, 2013.

3. Ji M, Liu Y, Li Q, Li XD, Zhao WQ, Zhang H, Zhang X, Jiang JT and $\mathrm{Wu}$ CP: PD-1/PD-L1 pathway in non-small-cell lung cancer and its relation with EGFR mutation. J Transl Med 13: 5, 2015.

4. Kim MY, Koh J, Kim S, Go H, Jeon YK and Chung DH: Clinicopathological analysis of PD-L1 and PD-L2 expression in pulmonary squamous cell carcinoma: Comparison with tumorinfiltrating $\mathrm{T}$ cells and the status of oncogenic drivers. Lung Cancer 88: 24-33, 2015.

5. Rosell R, Carcereny E, Gervais R, Vergnenegre A, Massuti B, Felip E, Palmero R, Garcia-Gomez R, Pallares C, Sanchez JM, et al; Spanish Lung Cancer Group in collaboration with Groupe Français de Pneumo-Cancérologie and Associazione Italiana Oncologia Toracica: Erlotinib versus standard chemotherapy as first-line treatment for European patients with advanced EGFR mutation-positive non-small-cell lung cancer (EURTAC): A multicentre, open-label, randomised phase 3 trial. Lancet Oncol 13: 239-246, 2012

6. Lee JK, Hahn S, Kim DW, Suh KJ, Keam B, Kim TM, Lee SH and Heo DS: Epidermal growth factor receptor tyrosine kinase inhibitors vs conventional chemotherapy in non-small cell lung cancer harboring wild-type epidermal growth factor receptor: A meta-analysis. JAMA 311: 1430-1437, 2014.

7. Cross DA, Ashton SE, Ghiorghiu S, Eberlein C, Nebhan CA, Spitzler PJ, Orme JP, Finlay MR, Ward RA, Mellor MJ, et al: AZD9291, an irreversible EGFR TKI, overcomes T790Mmediated resistance to EGFR inhibitors in lung cancer. Cancer Discov 4: 1046-1061, 2014.

8. Akbay EA, Koyama S, Carretero J, Altabef A, Tchaicha JH, Christensen CL, Mikse OR, Cherniack AD, Beauchamp EM, Pugh TJ, et al: Activation of the PD-1 pathway contributes to immune escape in EGFR-driven lung tumors. Cancer Discov 3: 1355-1363, 2013.

9. Azuma K, Ota K, Kawahara A, Hattori S, Iwama E, Harada T, Matsumoto K, Takayama K, Takamori S, Kage M, et al: Association of PD-L1 overexpression with activating EGFR mutations in surgically resected nonsmall-cell lung cancer. Ann Oncol 25: 1935-1940, 2014.

10. Tang Y, Fang W, Zhang Y, Hong S, Kang S, Yan Y, Chen N, Zhan J, He X, Qin T, et al: The association between PD-L1 and EGFR status and the prognostic value of PD-L1 in advanced non-small cell lung cancer patients treated with EGFR-TKIs Oncotarget 6: 14209-14219, 2015.
11. D'Incecco A, Andreozzi M, Ludovini V, Rossi E, Capodanno A, Landi L, Tibaldi C, Minuti G, Salvini J, Coppi E, et al: PD-1 and PD-L1 expression in molecularly selected non-small-cell lung cancer patients. Br J Cancer 112: 95-102, 2015.

12. Afreen S and Dermime S: The immunoinhibitory B7-H1 molecule as a potential target in cancer: Killing many birds with one stone. Hematol Oncol Stem Cell Ther 7: 1-17, 2014.

13. Muenst S, Soysal SD, Tzankov A and Hoeller S: The PD-1/ PD-L1 pathway: Biological background and clinical relevance of an emerging treatment target in immunotherapy. Expert Opin Ther Targets 19: 201-211, 2015.

14. Sharma P and Allison JP: The future of immune checkpoint therapy. Science 348: 56-61, 2015.

15. Wölfle SJ, Strebovsky J, Bartz H, Sähr A, Arnold C, Kaiser C, Dalpke AH and Heeg K: PD-L1 expression on tolerogenic APCs is controlled by STAT-3. Eur J Immunol 41: 413-424, 2011.

16. Alvarez JV, Greulich H, Sellers WR, Meyerson M and Frank DA: Signal transducer and activator of transcription 3 is required for the oncogenic effects of non-small-cell lung cancer-associated mutations of the epidermal growth factor receptor. Cancer Res 66: 3162-3168, 2006.

17. Gao SP, Mark KG, Leslie K, Pao W, Motoi N, Gerald WL, Travis WD, Bornmann W, Veach D, Clarkson B, et al: Mutations in the EGFR kinase domain mediate STAT3 activation via IL-6 production in human lung adenocarcinomas. J Clin Invest 117: 3846-3856, 2007.

18. Cortez MA, Ivan C, Valdecanas D, Wang X, Peltier HJ, Ye Y, Araujo L, Carbone DP, Shilo K, Giri DK, et al: PDL1 regulation by p53 via miR-34. J Natl Cancer Inst 108: 108, 2015.

19. Scaltriti $M$ and Baselga $J$ : The epidermal growth factor receptor pathway: A model for targeted therapy. Clin Cancer Res 12: 5268-5272, 2006.

20. Keedy VL, Temin S, Somerfield MR, Beasley MB, Johnson DH, McShane LM, Milton DT, Strawn JR, Wakelee HA and Giaccone G: American Society of Clinical Oncology provisional clinical opinion: Epidermal growth factor receptor (EGFR) Mutation testing for patients with advanced non-small-cell lung cancer considering first-line EGFR tyrosine kinase inhibitor therapy. J Clin Oncol 29: 2121-2127, 2011.

21. Lee SJ, Jang BC, Lee SW, Yang YI, Suh SI, Park YM, Oh S, Shin JG, Yao S, Chen L, et al: Interferon regulatory factor-1 is prerequisite to the constitutive expression and IFN-gammainduced upregulation of B7-H1 (CD274). FEBS Lett 580: 755-762, 2006.

22. Velcheti V, Schalper KA, Carvajal DE, Anagnostou VK, Syrigos KN, Sznol M, Herbst RS, Gettinger SN, Chen L and Rimm DL: Programmed death ligand-1 expression in non-small cell lung cancer. Lab Invest 94: 107-116, 2014.

23. Barber DL, Wherry EJ, Masopust D, Zhu B, Allison JP, Sharpe AH, Freeman GJ and Ahmed R: Restoring function in exhausted CD8 T cells during chronic viral infection. Nature 439: 682-687, 2006.

24. Abiko K, Matsumura N, Hamanishi J, Horikawa N, Murakami R, Yamaguchi K, Yoshioka Y, Baba T, Konishi I and Mandai M: IFN- $\gamma$ from lymphocytes induces PD-L1 expression and promotes progression of ovarian cancer. Br J Cancer 112: 1501-1509, 2015.

25. Ritprajak P and Azuma M: Intrinsic and extrinsic control of expression of the immunoregulatory molecule PD-L1 in epithelial cells and squamous cell carcinoma. Oral Oncol 51: 221-228, 2015.

26. Taube JM, Anders RA, Young GD, Xu H, Sharma R, McMiller TL, Chen S, Klein AP, Pardoll DM, Topalian SL, et al: Colocalization of inflammatory response with B7-h1 expression in human melanocytic lesions supports an adaptive resistance mechanism of immune escape. Sci Transl Med 4: 127ra37, 2012.

27. Liu J, Hamrouni A, Wolowiec D, Coiteux V, Kuliczkowski K, Hetuin D, Saudemont A and Quesnel B: Plasma cells from multiple myeloma patients express B7-H1 (PD-L1) and increase expression after stimulation with IFN-\{gamma\} and TLR ligands via a MyD88-, TRAF6-, and MEK-dependent pathway. Blood 110: 296-304, 2007.

28. Atefi M, Avramis E, Lassen A, Wong DJ, Robert L, Foulad D, Cerniglia M, Titz B, Chodon T, Graeber TG, et al: Effects of MAPK and PI3K pathways on PD-L1 expression in melanoma. Clin Cancer Res 20: 3446-3457, 2014. 
29. Parsa AT, Waldron JS, Panner A, Crane CA, Parney IF, Barry JJ, Cachola KE, Murray JC, Tihan T, Jensen MC, et al: Loss of tumor suppressor PTEN function increases B7-H1 expression and immunoresistance in glioma. Nat Med 13: 84-88, 2007.

30. Xu C, Fillmore CM, Koyama S, Wu H, Zhao Y, Chen Z, HerterSprie GS, Akbay EA, Tchaicha JH, Altabef A, et al: Loss of Lkb1 and Pten leads to lung squamous cell carcinoma with elevated PD-L1 expression. Cancer Cell 25: 590-604, 2014.

31. Lin K, Cheng J, Yang T, Li Y and Zhu B: EGFR-TKI downregulates PD-L1 in EGFR mutant NSCLC through inhibiting NF-кB. Biochem Biophys Res Commun 463: 95-101, 2015.

32. Zhang Y, Huang S, Gong D, Qin Y and Shen Q: Programmed death-1 upregulation is correlated with dysfunction of tumorinfiltrating $\mathrm{CD}^{+} \mathrm{T}$ lymphocytes in human non-small cell lung cancer. Cell Mol Immunol 7: 389-395, 2010.
33. Chen YB, Mu CY and Huang JA: Clinical significance of programmed death-1 ligand-1 expression in patients with non-small cell lung cancer: A 5-year-follow-up study. Tumori 98: 751-755, 2012.

34. Mu CY, Huang JA, Chen Y, Chen C and Zhang XG: High expression of PD-L1 in lung cancer may contribute to poor prognosis and tumor cells immune escape through suppressing tumor infiltrating dendritic cells maturation. Med Oncol 28: 682-688, 2011.

35. Robert L, Ribas A and Hu-Lieskovan S: Combining targeted therapy with immunotherapy. Can $1+1$ equal more than 2 ? Semin Immunol 28: 73-80, 2016.

36. Wargo JA, Cooper ZA and Flaherty KT: Universes collide: Combining immunotherapy with targeted therapy for cancer. Cancer Discov 4: 1377-1386, 2014. 